\title{
Validity of the diagnostic criteria for chronic cerebrospinal venous insufficiency and association with multiple sclerosis
}

\author{
Fiona Costello MD, Jayesh Modi MD, David Lautner MD, Deepak Bhayana MD, James N. Scott MD, \\ W. Jeptha Davenport MD, Jessie Trufyn BSc MSc, Richard Frayne PhD, Viesha A. Ciura MD, \\ Mayank Goyal MD, Jean Mah MD, Michael D. Hill MD MSc
}

Competing interests: See end of article.

This article has been peer reviewed.

Correspondence to:

Fiona Costello,

fionacostello@rogers.com

CMAJ 2014. DOI:10.1503 /cmaj.131431

\begin{abstract}
Background: The chronic cerebrospinal venous insufficiency theory proposes that altered cerebral venous hemodynamics play a role in the pathophysiology of multiple sclerosis. We aimed to explore the validity of this hypothesis by assessing the diagnostic criteria for chronic cerebrospinal venous insufficiency in persons with and without multiple sclerosis.
\end{abstract}

Methods: We compared the proportion of venous outflow abnormalities between patients with multiple sclerosis and healthy controls using extracranial Doppler ultrasonography and gadolinium-enhanced magnetic resonance venography. Interpreting radiologists were blinded to the clinical status of participants.

Results: We enrolled 120 patients with multiple sclerosis and 60 healthy controls. High proportions of both patients $(67 / 115$ [58\%]) and con- trols $(38 / 60[63 \%])$ met 1 or more of the proposed ultrasound criteria for diagnosis of chronic cerebrospinal venous insufficiency $(p=0.6)$. A minority of patients (23/115 [20\%]) and controls $(6 / 60$ [10\%]) fulfilled 2 or more of the proposed criteria $(p=0.1)$. There were no differences between patients and controls in the prevalence of each individual ultrasound criterion. Similarly, there were no differences in intracranial or extracranial venous patency between groups, as measured by magnetic resonance venography.

Interpretation: We detected no differences in the proportion of venous outflow abnormalities between patients with multiple sclerosis and healthy controls. Moreover, our study revealed significant methodologic concerns regarding the proposed diagnostic criteria for chronic cerebrospinal venous insufficiency that challenge their validity.
$\mathrm{M}$ ultiple sclerosis is an inflammatory disease of the central nervous system, believed to arise from a dysfunctional immune-mediated response in a genetically susceptible host. ${ }^{1}$ In 2009, "chronic cerebrospinal venous insufficiency" was proposed to play an etiologic role in multiple sclerosis. ${ }^{2-4}$ Despite an abundance of published literature on this topic, ${ }^{2-28}$ a causal link has not been established. Recent meta-analyses have suggested a strong association between an ultrasound-based diagnosis of chronic cerebrospinal venous insufficiency and multiple sclerosis, ${ }^{26,28}$ yet there has been significant heterogeneity across studies. ${ }^{26,27}$ A factor contributing to this heterogeneity appears to be the involvement of investigators who support endovascular procedures as a treatment for multiple sclerosis. ${ }^{27}$ Furthermore, these meta-analyses have been predicated on the assumption that valid diagnostic criteria for chronic cerebrospinal venous insufficiency exist.

We aimed to explore the validity of the chronic cerebrospinal venous insufficiency theory by using extracranial ultrasonography and gadoliniumenhanced magnetic resonance venography to compare the proportion of venous outflow abnormalities between patients with multiple sclerosis and healthy individuals. Our primary hypothesis was that if chronic cerebrospinal venous insufficiency is associated with multiple sclerosis, we would detect significant evidence of venous outflow obstruction in patients relative to controls.

\section{Methods}

\section{Study design}

This cross-sectional study involved participants who were evaluated at the University of Calgary. The protocol was approved by the institution's Conjoint Health Research Ethics Board, and participants provided written, informed consent.

\section{Study population}

Hundreds of patients followed at the Calgary Multiple Sclerosis Clinic volunteered to participate in the study. We consecutively screened and enrolled individuals from this group who met the inclusion 
criteria until we reached predetermined quotas for each subtype of multiple sclerosis. We recruited age-matched (by decile) and sex-matched controls from volunteers who expressed interest in the study. Eligible patients had clinically isolated syndromes, ${ }^{29}$ relapsing-remitting multiple sclerosis proven by McDonald ${ }^{29}$ and $\operatorname{Poser}^{30}$ criteria, secondary progressive multiple sclerosis, primary progressive multiple sclerosis ${ }^{29,31}$ or antibody-positive neuromyelitis optica. ${ }^{32}$ Participants provided consent themselves or with the aid of guardian. Exclusion criteria are described in Appendix 1, available at www.cmaj.ca/lookup/suppl/doi:10.1503/cmaj .131431/-/DC1.

\section{Screening}

Participants underwent a standardized clinical interview and data collection at baseline (Figure 1). This phase served to establish the diagnosis and confirm eligibility for all participants.

\section{Clinical assessment}

All participants underwent grading according to the Expanded Disability Status Scale. ${ }^{33}$ This grading was performed by a neurologist (F.C., W.J.D. or J. Mah) blinded to the imaging results.

\section{Ultrasound procedures}

Certified technologists ${ }^{34}$ blinded to the clinical status of participants performed the ultrasonography, using a Phillips IU-22 unit, with a linear-array, broad-bandwidth 9-3 $\mathrm{MHz}$ transducer on the manufacturer's setting for "upper extremity venous." Technical parameters were optimized for accurate results. Scanning was performed with participants in the supine and then sitting positions. Participants were instructed to breathe normally and to avoid performing a Valsalva manoeuvre.

The internal jugular and vertebral veins were assessed with grey-scale (B-mode) imaging in both longitudinal and transverse planes. Standardized measurements of each vein included vessel diameter and cross-sectional area. Greyscale analysis of the internal jugular valve was performed in 2 planes to look for anomalies. When valve identification was challenging with the linear-array probe, a curvilinear-array probe with a small footprint was used.

We assessed venous patency using colour Doppler sonography. Pulsed-wave analysis documented flow direction, velocity and volume. We considered spectral and colour Doppler sonography results to be abnormal if flow appeared to be accelerated or if the normal variability of venous waveforms was lost. Colour Doppler sonography in the longitudinal plane was performed in the lower internal segment of the jugular vein, just above the level of the valve. If reflux was detected, a pulsed-wave spectral tracing was performed to measure duration. The cross-sectional area was viewed in grey scale in the transverse plane, with measurement at the superior, inferior and mid sec-

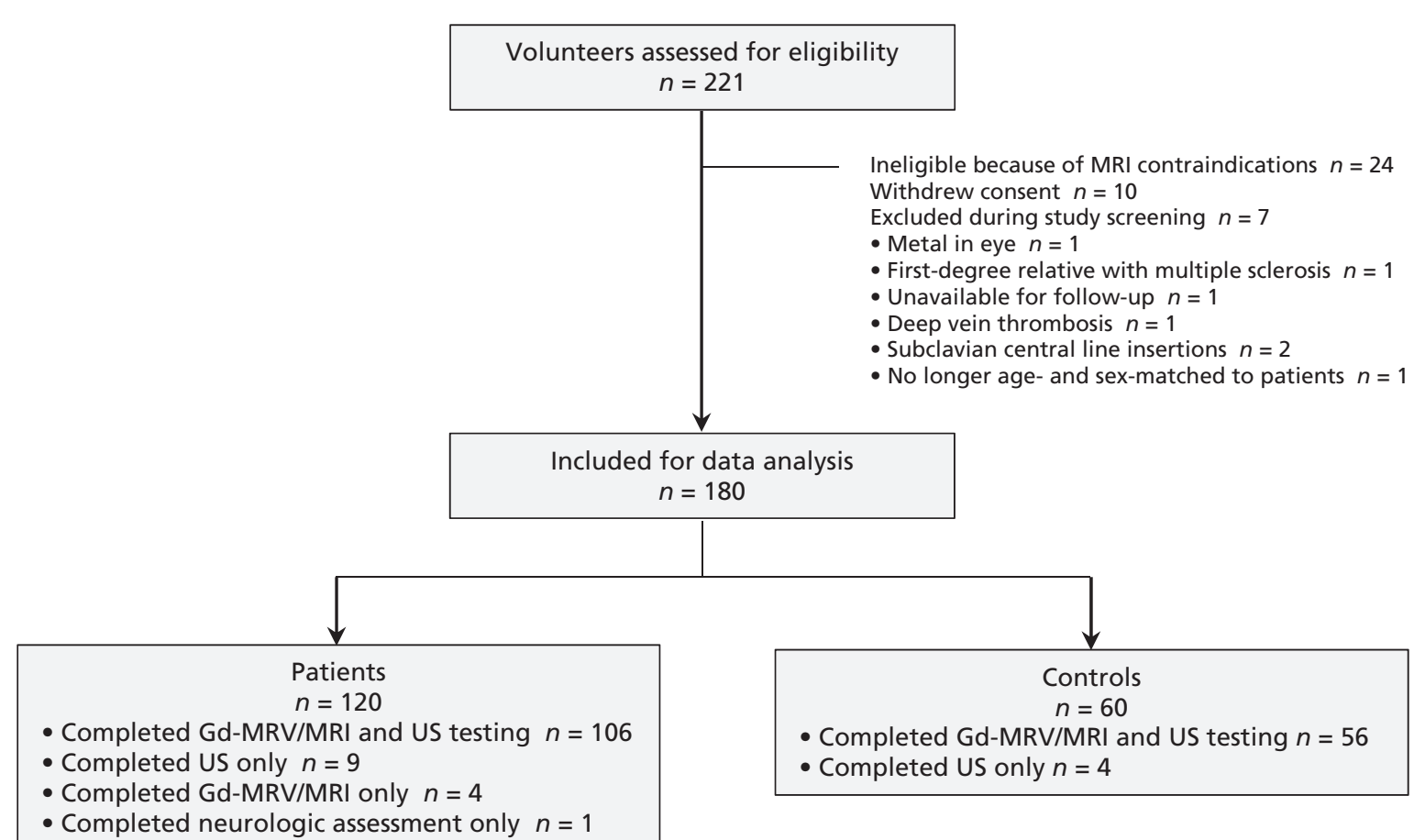

Figure 1: Enrolment of patients with multiple sclerosis and healthy controls, reasons for exclusions and types of data obtained. Gd = gadolinium, MRI = magnetic resonance imaging, $M R V=$ magnetic resonance venography, US = ultrasonography. 
tions of the internal jugular veins, with the participant in the supine and sitting positions.

\section{Ultrasound criteria}

Two radiologists (D.L, D.B.), who were blinded to participants' clinical status and magnetic resonance imaging results, interpreted the ultrasound results. They determined the presence of obstruction of venous outflow using the following criteria proposed for diagnosis of chronic cerebrospinal venous insufficiency ${ }^{3,4}$ (excluding reflux in deep cerebral veins, because we did not perform transcranial ultrasonography):

1. Reflux $>0.88$ seconds in the internal jugular and vertebral veins (sitting or supine)

2. B-mode evidence of stenosis of the internal jugular vein, defined as local reduction of cross-sectional area $<50 \%$ or $\leq 0.3 \mathrm{~cm}^{2}$ in the supine position

3. Flow not Doppler-detectable in the internal jugular and vertebral veins

Table 1: Characteristics of study participants

No. (\%) of participants*

\begin{tabular}{ccc} 
& \multicolumn{2}{c}{ No. (\%) of participants* } \\
\cline { 2 - 3 } Characteristic & Patients & Controls \\
& $n=120$ & $n=60$
\end{tabular}

\section{Demographic}

\begin{tabular}{lcc} 
Age, yr, mean \pm SD & $45.7 \pm 11.9$ & $45.3 \pm 11.2$ \\
Sex, female & $89(74)$ & $45(75)$ \\
\hline Ethnic origin, white & $110(92)$ & $56(93)$
\end{tabular}

\section{Clinical factors}

Family history of MS

Duration of MS, yr, median (IQR)

EDSS, median (IQR)

\section{MS subtype}

$\begin{array}{lrl}\text { Relapsing-remitting } & 86(72) & \text { NA } \\ \text { Secondary progressive } & 17(14) & \text { NA } \\ \text { Primary progressive } & 12(10) & \text { NA } \\ \text { Clinically isolated syndrome } & 4(3) & \text { NA } \\ \text { Neuromyelitis optica } & 1(1) & \text { NA }\end{array}$

\section{Current disease-modifying} treatment

\begin{tabular}{|lcl|}
\hline None & $58(48)$ & NA \\
\hline Glatiramer acetate (Copaxone) & $42(35)$ & NA \\
\hline Interferon ß1a (Rebif) & $12(10)$ & NA \\
Interferon B1a (Avonex) & $3(2)$ & NA \\
\hline Interferon B1b (Betaseron) & $3(2)$ & NA \\
\hline Fingolimod (Gilenya) & $2(2)$ & NA \\
\hline
\end{tabular}

Note: EDSS = Expanded Disability Status Scale (where steps 1.0-4.5 refer to patients with MS who are fully ambulatory, steps 5.0-9.5 are defined by impairment in neurologic function with progressive disability corresponding to higher numbers on the scale, and step 10.0 refers to death due to $M S)^{33}, I Q R=$ interquartile range, $M S=$ multiple sclerosis, $N A=$ not applicable, SD = standard deviation

*Except where indicated otherwise.
4. Reverted postural control of the main cerebral venous outflow

\section{Magnetic resonance venography: procedures and criteria}

We performed gadolinium-enhanced magnetic resonance venography according to the procedures described in Appendix 1 (available at www.cmaj.ca/lookup/suppl/doi:10.1503/cmaj.13 1431/-/DC1). At the start of this study, there were no established criteria to diagnose chronic cerebrospinal venous insufficiency by this method. Consequently, we evaluated extracranial venous outflow and burden of disease according to the criteria presented in Appendix 1. Three radiologists (J. Modi, J.N.S. or M.G.), blinded to participants' clinical status and ultrasound results, interpreted these results.

\section{Statistical analyses}

The primary objective of the study was to determine the proportion of patients and controls with ultrasound and magnetic resonance venography evidence of venous outflow obstruction. Secondary outcomes included magnetic resonance imaging measures of brain inflammation and Expanded Disability Status Scale scores. The selection of 120 patients and 60 controls was a sample of convenience because the rate of venous outflow obstruction in patients versus controls in a Canadian population is unknown. The primary analysis compared the number of patients with venous outflow obstruction as determined by ultrasound and gadoliniumenhanced magnetic resonance venography versus controls with McNemar's test.

\section{Results}

\section{Demographic characteristics}

After screening, 180 participants were included in the study (Figure 1). Among the 120 patients, multiple sclerosis had been present for a median duration of 10.5 years and was predominantly of the relapsing-remitting subtype (86 patients [72\%]) (Table 1).

\section{Ultrasound results}

Evaluations of reflux, cross-sectional area and velocity of internal jugular venous flow illustrated variable measurements that did not differ between groups (Appendix 2, available at www.cmaj .ca/lookup/suppl/doi:10.1503/cmaj.131431/-/DC1). A high proportion of patients $(67 / 115$ [58\%]) and controls $(38 / 60$ [63\%]) met 1 or more of the proposed criteria for the diagnosis of chronic cerebrospinal venous insufficiency, ${ }^{4}$ with no differences between groups $(p=0.6)$ (Table 2$)$. A minority of 
patients (23/115 [20\%]) and controls (6/60 [10\%]) fulfilled 2 or more diagnostic criteria ${ }^{4}(p=0.1)$ (Table 2). Notably, baseline characteristics did not differ for these participants relative to those who fulfilled fewer criteria (Appendix 3, available at www.cmaj.ca/lookup/suppl/doi:10.1503/cmaj $.131431 /-/ D C 1)$. When we analyzed the results for individual ultrasound criteria, we found no differences between patients and controls (Table 2).
Magnetic resonance venography results

Patients showed greater "lesional" burden than controls. Lesions were detected in controls but were not accompanied by brain atrophy or gadolinium enhancement suggestive of underlying demyelination (Table 3). Post hoc analysis showed that these nonspecific lesions were fewer in number (relative to lesions observed in patients), and they were considered age-appro-

Table 2: Ultrasound criteria for chronic cerebrospinal vascular insufficiency in patients with multiple sclerosis and healthy controls

\begin{tabular}{|c|c|c|c|c|}
\hline \multirow[b]{2}{*}{ Criterion* } & \multicolumn{2}{|c|}{ No. (\%) of participantst } & \multirow[b]{2}{*}{$p$ value } & \multirow[b]{2}{*}{ OR $(95 \% \mathrm{Cl}) \ddagger$} \\
\hline & $\begin{array}{l}\text { Patients } \\
n=115\end{array}$ & $\begin{array}{c}\text { Controls } \\
n=60\end{array}$ & & \\
\hline $\begin{array}{l}\text { Criterion 1: reflux (IJV, VV: right and } \\
\text { left, upright and supine) }\end{array}$ & & & 0.3 & \\
\hline None & $68(59)$ & $30(50)$ & & \\
\hline 1 vein & $25(22)$ & $22(37)$ & & \\
\hline 2 veins & $11(10)$ & $5(8)$ & & \\
\hline 3 veins & $9(8)$ & $3(5)$ & & \\
\hline 4 veins & $2(2)$ & $0 \quad(0)$ & & \\
\hline Criterion 3: IJV stenosis (right and left) & & & 0.4 & \\
\hline Both normal & $89(77)$ & $52(87)$ & & \\
\hline 1 abnormal & $17(15)$ & $6(10)$ & & \\
\hline 2 abnormal & $9(8)$ & $2(3)$ & & \\
\hline $\begin{array}{l}\text { Criterion 4: no Doppler-detectable } \\
\text { flow (IJV, VV: right and left, upright } \\
\text { and supine) }\end{array}$ & & & $>0.9$ & \\
\hline Normal & $113(98)$ & $59(98)$ & & \\
\hline 1 vein & $2(2)$ & $1(2)$ & & \\
\hline $\begin{array}{l}\text { Criterion 5: negative CSA in IJV (right } \\
\text { and left) }\end{array}$ & & & 0.2 & \\
\hline Change in CSA $>0$ & $94(82)$ & $54(90)$ & & \\
\hline Change in $\mathrm{CSA} \leq 0$ & $21(18)$ & $6(10)$ & & \\
\hline \multicolumn{5}{|l|}{ No. of ultrasound criteria met } \\
\hline$\geq 1$ & $67(58)$ & $38(63)$ & 0.6 & $0.9(0.4-1.6)$ \\
\hline$\geq 2$ & $23(20)$ & $6(10)$ & 0.1 & $2.2(0.8-7.2)$ \\
\hline$\geq 3$ & $6(5)$ & $1(2)$ & 0.5 & $3.2(0.3-151.8)$ \\
\hline$\geq 4$ & $0 \quad(0)$ & $0 \quad(0)$ & NA & NA \\
\hline 0 & $48(42)$ & $22(37)$ & 0.6 & \\
\hline Exactly 1 & $44(38)$ & $32(53)$ & 0.08 & \\
\hline Exactly 2 & $17(15)$ & $5(8)$ & 0.3 & \\
\hline Exactly 3 & $6(5)$ & $1(2)$ & 0.4 & \\
\hline Exactly 4 & $0 \quad(0)$ & $0 \quad(0)$ & NA & \\
\hline \multicolumn{5}{|c|}{$\begin{array}{l}\text { Note: } \mathrm{Cl}=\text { confidence interval, CSA = cross-sectional area, IJV = internal jugular vein, NA = not applicable, OR = odds ratio, VV = } \\
\text { vertebral vein } \\
\text { *All criteria as presented by Zamboni and colleagues., }{ }^{3,4} \text { Criterion } 1=\text { reflux in the IJVs and/or VVs in sitting or supine position, } \\
\text { defined as reflux }>0.88 \mathrm{~s} \text {; criterion } 3=\text { Doppler B-mode evidence of IJV stenosis, defined as local reduction of cross-sectional } \\
\text { area }<50 \% \text { and/or } \leq 0.3 \mathrm{~cm}^{2} \text { in supine position; criterion } 4=\text { flow not Doppler-detectable in the IJVs and/or VVs; criterion } 5, \\
\text { reverted postural control of the main cerebral venous outflow. For the current study, criterion } 2 \text { (transcranial Doppler } \\
\text { assessment of internal cerebral veins) was not performed. } \\
\text { tExcept where indicated otherwise. } \\
\text { tFisher's exact test for patients and controls meeting } 1 \text { or more US criteria, } 2 \text { or more US criteria, } 3 \text { or more US criteria, or } 4 \text { or } \\
\text { more US criteria. }\end{array}$} \\
\hline
\end{tabular}


Table 3: Results of magnetic resonance imaging and gadoliniun-enhanced venography

\begin{tabular}{|c|c|c|c|c|c|c|c|c|c|}
\hline \multirow[b]{2}{*}{ MR result } & \multicolumn{8}{|c|}{ No. (\%) of participants } & \multirow[b]{2}{*}{$p$ value } \\
\hline & \multicolumn{4}{|c|}{$\begin{array}{l}\text { Patients } \\
n=110\end{array}$} & \multicolumn{4}{|c|}{$\begin{array}{c}\text { Controls } \\
n=56\end{array}$} & \\
\hline \multicolumn{10}{|l|}{ MR imaging } \\
\hline \multicolumn{10}{|l|}{ No. of white matter lesions } \\
\hline 0 & \multicolumn{4}{|c|}{$5 \quad(5)$} & \multicolumn{4}{|c|}{$29(52)$} & \multirow[t]{4}{*}{$<0.001$} \\
\hline $1-10$ & \multicolumn{4}{|c|}{$34(31)$} & \multicolumn{4}{|c|}{$22(39)$} & \\
\hline $11-20$ & \multicolumn{4}{|c|}{$33(30)$} & \multicolumn{4}{|c|}{5 (9) } & \\
\hline $21-30$ & \multicolumn{4}{|c|}{$38(35)$} & \multicolumn{4}{|c|}{$0 \quad(0)$} & \\
\hline Enhancing lesions & \multicolumn{4}{|c|}{$12(11)$} & \multicolumn{4}{|c|}{$0(0)$} & 0.2 \\
\hline Parenchymal atrophy & & & & & & & & & $<0.001$ \\
\hline Corpus callosum atrophy & & & & & & & & & $<0.001$ \\
\hline MR venography (TRICKS)* & & ht & Le & & Rig & & Le & & Right / Left \\
\hline IJV dominance & & (26) & & (11) & 12 & (21) & 3 & (5) & $0.3 / 0.3$ \\
\hline IJV flattening C1-C3 level, \% & $n=$ & 109 & $n=$ & 109 & & & & & \\
\hline $0-24$ & 32 & (29) & 32 & (29) & 14 & (25) & 17 & (30) & $0.9 / 0.7$ \\
\hline $25-49$ & 44 & $(40)$ & 42 & (39) & 23 & (41) & 17 & (30) & \\
\hline $50-74$ & 10 & (9) & 11 & $(10)$ & 5 & (9) & 6 & (11) & \\
\hline $75-100$ & 23 & (21) & 24 & $(22)$ & 14 & (25) & 16 & (29) & \\
\hline IJV flattening C4-C5 level, \% & & & & & & & & & \\
\hline $0-24$ & 94 & (86) & 87 & $(80)$ & 51 & (91) & 46 & (82) & $0.9 / 0.8$ \\
\hline $25-49$ & 5 & (5) & 9 & (8) & 1 & (2) & 5 & (9) & \\
\hline $50-74$ & 3 & (3) & 1 & $(1)$ & 1 & (2) & 1 & (2) & \\
\hline $75-100$ & 7 & (6) & 12 & (11) & 3 & (5) & 4 & (7) & \\
\hline IJV flattening C6-T1 level, \% & & & & & & & & & \\
\hline $0-24$ & 55 & $(50)$ & 87 & $(80)$ & 29 & (52) & 46 & (82) & $0.9 / 0.9$ \\
\hline $25-49$ & 21 & (19) & 9 & (8) & 11 & (20) & 5 & (9) & \\
\hline $50-74$ & 8 & (7) & 1 & $(1)$ & 2 & (4) & 1 & (2) & \\
\hline $75-100$ & 25 & (23) & 12 & $(11)$ & 14 & (25) & 4 & (7) & \\
\hline Collateral vein score & & & & & & & & & \\
\hline Normal & 25 & (23) & 25 & $(23)$ & 12 & (21) & 13 & (23) & $>0.9 / 0.8$ \\
\hline Mild & 45 & (41) & 39 & (36) & 25 & (45) & 23 & (41) & \\
\hline Moderate & 29 & (27) & 37 & (34) & 14 & (25) & 15 & (27) & \\
\hline Prominent & 10 & (9) & 8 & (7) & 5 & (9) & 5 & (9) & \\
\hline Intracranial venous stenosis & 0 & (0) & & & 0 & (0) & & & NA \\
\hline Transverse sinus dominance & 23 & (21) & 12 & $(11)$ & 13 & (23) & 4 & (7) & $0.4 / 0.8$ \\
\hline MR pre/post Gd RAGE & & & & & & & & & \\
\hline IJV narrowing ${ }^{\prime}$ at $\mathrm{C} 1-\mathrm{C} 3$ level & & & & & & & & & \\
\hline $0-24$ & 35 & $(32)$ & 38 & (35) & 14 & (25) & 17 & (30) & $0.8 / 0.4$ \\
\hline $25-49$ & 44 & (40) & 43 & (39) & 25 & (45) & 18 & (32) & \\
\hline $50-74$ & 9 & (8) & 11 & $(10)$ & 4 & (7) & 6 & (11) & \\
\hline $75-100$ & 21 & (19) & 17 & $(16)$ & 13 & (23) & 15 & (27) & \\
\hline IJV narrowingt at C4-C6 level & & & & & & & & & \\
\hline $0-24$ & 103 & (94) & 98 & $(90)$ & 55 & (98) & 49 & (88) & $0.8 / 0.9$ \\
\hline $25-49$ & 5 & (5) & 7 & (6) & 1 & (2) & 5 & & \\
\hline $50-74$ & 1 & (1) & 1 & (1) & 0 & (0) & 0 & (0) & \\
\hline $75-100$ & 0 & (0) & 3 & (3) & 0 & (0) & 2 & (4) & \\
\hline IJV narrowingt at C7-T1 level & & & & & & & & & \\
\hline $0-24$ & & (56) & 69 & (63) & 31 & (55) & 32 & (57) & $0.7 / 0.7$ \\
\hline $25-49$ & 21 & (19) & 11 & $(10)$ & 10 & (18) & 8 & (14) & \\
\hline $50-74$ & 8 & (7) & & (7) & 2 & (4) & 3 & (5) & \\
\hline $75-100$ & 19 & (17) & 21 & (19) & 13 & (23) & 13 & (23) & \\
\hline Mass or lymph nodes & 0 & $(0)$ & & & 0 & (0) & & & NA \\
\hline $\begin{array}{l}\text { Note: } \mathrm{Gd}=\text { gadolinium, IJV = } \\
\text { TRICKS = time-resolved imagi } \\
\text { *For one patient, some MR ve } \\
\text { †Narrowing commonly by ext }\end{array}$ & $n$ & $\begin{array}{l}\text { AR }=\mathrm{m} \\
\text { vere ur } \\
\mathrm{n} \text { the } \mathrm{t}\end{array}$ & 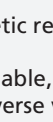 & $\begin{array}{l}\text { onan } \\
\text { so da } \\
\text { serteb }\end{array}$ & 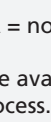 & $\begin{array}{l}\text { t appl } \\
\text { ilable }\end{array}$ & $\begin{array}{l}\text { ole, RAC } \\
\text { only } 10\end{array}$ & $\begin{array}{l}\mathrm{E}=\mathrm{ra} \\
9 \text { pati }\end{array}$ & gradient echo \\
\hline
\end{tabular}


priate for the controls. ${ }^{1}$ There were no differences in measures of venous outflow between patients and controls (Table 3).

\section{Interpretation}

We observed no differences in the proportion of venous outflow abnormalities, as measured by ultrasonography or gadolinium-enhanced magnetic resonance venography, between patients with multiple sclerosis and healthy controls.

Previously, Zamboni and colleagues ${ }^{3,4}$ reported that diagnosis of chronic cerebrospinal venous insufficiency required fulfillment of at least 2 of 5 ultrasound criteria. They detected greater prevalence of several criteria among patients than among controls: reflux (71\% v. 0\%), B-mode evidence of internal jugular vein stenosis $(37 \% \mathrm{v}$. $0 \%$ ), absent flow detectable by Doppler ultrasonography in the internal jugular or vertebral veins $(52 \%$ v. $3 \%)$ and reversed postural flow in the internal jugular vein $(55 \%$ v. $11 \%){ }^{3}$ When these criteria were applied in the evaluation of 109 patients with multiple sclerosis and 177 controls, each patient was deemed to meet at least 2 criteria, whereas none of the control participants did so. ${ }^{4}$ The sensitivity, specificity, and positive and negative predictive values for the proposed criteria were each $100 \% .{ }^{4}$ Numerous groups have since been unable to replicate these findings. ${ }^{5-}$ 8,13,16,19,21 Doepp and associates ${ }^{5}$ reported that no patients with multiple sclerosis and no controls fulfilled more than 1 ultrasound criterion for chronic cerebrospinal venous insufficiency. Baretto and colleagues ${ }^{21}$ noted that $30 \%$ of their study's participants fulfilled 1 criterion, $5 \%$ met 2 criteria, and none satisfied more than 2 criteria for the diagnosis. The diagnostic criteria proposed for chronic cerebrospinal venous insufficiency are overly inclusive and nonspecific. This would explain why the condition was detected in $56 \%$ of patients with multiple sclerosis and $23 \%$ of controls in a prior study. ${ }^{9}$ It also accounts for our observation that $10 \%$ of the normal population would apparently carry the diagnosis.

The lack of reproducibility of the original findings reported by Zamboni and colleagues ${ }^{3,4}$ may relate to "methodological flaws" and the "pathophysiologic implausibility" of the ultrasound features used to define chronic cerebrospinal venous insufficiency. ${ }^{20}$ The definition of venous reflux used by Zamboni and colleagues has been challenged because spectral Doppler measurements of duration were not implemented..$^{21}$ Furthermore, the 0.88 -second threshold used to identify reflux was adopted from a study that examined internal jugular vein valve insufficiency during the Valsalva manoeuvre and was not based on validated methodol- ogy. ${ }^{35}$ Our findings indicate that the phenomenon characterized as "reflux" in the proposed criteria consists of low-velocity retrograde flow, which is almost exclusively observed in the periphery of the inferior internal jugular vein, near its confluence with the valve or subclavian vein. This flow was frequently present at a length of less than $2 \mathrm{~cm}$, was of small volume and did not reach a height above the thyroid cartilage in any participant. We observed no evidence of large-volume reflux that would have approached the intracranial veins. Therefore, the phenomenon termed "reflux" was trivial in our observations and likely represented an eddy current near recesses associated with the valves of the inferior jugular vein (Video 1). Because this finding is nonpathologic, it is not surprising that "reflux" was frequently detected in both controls (50\%) and patients with multiple sclerosis $(41 \%)$.

In the proposed criteria, ${ }^{3,4}$ venous "stenosis" was defined as a $50 \%$ reduction in the crosssectional area of the internal jugular vein or a value less than or equal to $0.3 \mathrm{~cm}^{2} .{ }^{3,4,20}$ Both these measures have subsequently been refuted on the basis of methodologic and anatomic considerations. ${ }^{20,21}$ The internal jugular vein is wider at its point of origin in the upper bulb and its confluence into the subclavian vein in the lower bulb. ${ }^{20}$ The cross-sectional area of the medium sections can be readily imaged using B-mode ultrasonography and varies with posture and central venous pressure. ${ }^{20}$ Given this inherent variability, defining internal jugular vein stenosis as a 50\% reduction is arbitrary. ${ }^{20}$ Similarly, the $0.3-\mathrm{cm}^{2}$ threshold was chosen on the basis of findings published by Lichtenstein and associates. ${ }^{36}$ Yet, in that same report, $23 \%$ of participants had a cross-sectional area less than or equal to $0.4 \mathrm{~cm}^{2}$, and measurements less than or equal to $0.3 \mathrm{~cm}^{2}$ were observed without associated pathology. ${ }^{20,36}$ Identifying a 50\% reduction in the cross-sectional area as evidence of internal jugular vein stenosis may lead to false-positive results, because the venous wall is thin and can easily be compressed, either

Please see the following video online at: www.cmaj.ca/lookup/suppl
/doi:10.1503/cmaj.131431/-/DC1.
Video 1 is an ultrasound video clip of the right internal jugular vein in
the sagittal plane obtained with colour flow Doppler in a control
participant. The video shows antegrade normal forward venous flow (toward
the heart), which appears blue and phasic in the vein lumen. There is an
intermittent small amount of peripheral red reversed flow against the deep vein
wall inferiorly. This observation reflects only a very small volume of reversed
blood flow, and the blood travels only a short distance (less than $1-2 \mathrm{~cm}$ ) from
the valve in the internal jugular vein in the root of the neck near the clavicle.
This trivial reversed flow is brief, occurs a fair distance (about $15 \mathrm{~cm}$ ) from the
brain and was seen commonly in control participants (normal volunteers,
without neurologic disease).


manually or by surrounding anatomic structures (Figure 2). The internal jugular vein is dilated at the craniocervical junction and distally, and it may appear narrowed within the region of the valves. We observed this phenomenon in our study, in which the overwhelming number of veins deemed to be "stenotic" 3,4 had an appearance suggesting a smoothly collapsed vein rather than a focal stricture. We did not observe focal vein narrowing with upstream dilatation, accompanied by a focal intraluminal source of accelerated flow and collateral formation, in any participant. The "stenoses" detected in our study would not have been identified as such by qualified ultrasonographers. This impression is supported by the fact that gadolinium-enhanced magnetic resonance venography showed no evidence of impaired cerebral venous drainage in any of our participants, despite the fact that 34 "stenotic" internal jugular veins were found with the proposed ultrasound criteria (Table 2).

We rarely observed "flow not Dopplerdetectable" in the internal jugular or vertebral veins of patients or controls. In the original work of Zamboni and colleagues, Doppler-detected flow was assessed "either with the pulse wave mode and the sample placed in the vessel at a 60 degree angle, or with the color coded mode." ${ }^{4}$ Veins were examined using "both the transversal and/or the longitudinal cervical access." ${ }^{4}$ These methods suggest insufficient attention to Doppler sonography techniques and physics. The presence of a cosine in the Doppler formula indicates that any time a structure is interrogated at an angle approaching $90^{\circ}$, the signal will diminish toward zero. Therefore, Doppler evaluation of a vessel in the transverse plane is apt to create a false-positive interpretation of absent flow. Several factors may have resulted in the reported absence of Doppler-detected flow in earlier pub- lications, including scanning without angle correction, failing to employ Doppler in a manner that kept the plane of imaging as parallel as possible to the direction of flow, and poor optimization of scale, gain and velocity settings.

\section{Limitations}

Perhaps the most striking finding in our study was the range of venous outflow anomalies detected that did not reflect pathology, but instead demonstrated the large natural variance in intracranial and extracranial venous anatomy (Figure 3). ${ }^{37}$ Venous capacitance vessels change appearance and flow depending upon patient position, intravascular volume status and ultrasonography technique. ${ }^{20}$ Given that venous walls are collapsible under conditions of reduced flow, the complexity of the extracranial venous circulation may be difficult to understand in any but a qualitative fashion. ${ }^{37}$ By extension, there are challenges in standardizing ultrasound examinations of the cerebral venous system. Our data indicate that venous peak velocity and cross-sectional area vary greatly with changes in posture.

We were unable to control for intravascular volume status, because invasive measurements of central venous pressure were not possible in this study. Many patients with multiple sclerosis manifest symptoms of bladder (detrusor) sphincter dyssynergia and may be reluctant to drink fluids, which may cause them to be relatively hypovolemic compared with controls. The effect of hydration on ultrasound findings in this patient population remains unclear.

In our study, magnetic resonance imaging was conducted with patients in the supine position only, because technology allowing upright scanning was not available. For this reason, the effects of posture on measures of venous patency could not be determined. Importantly, we noted
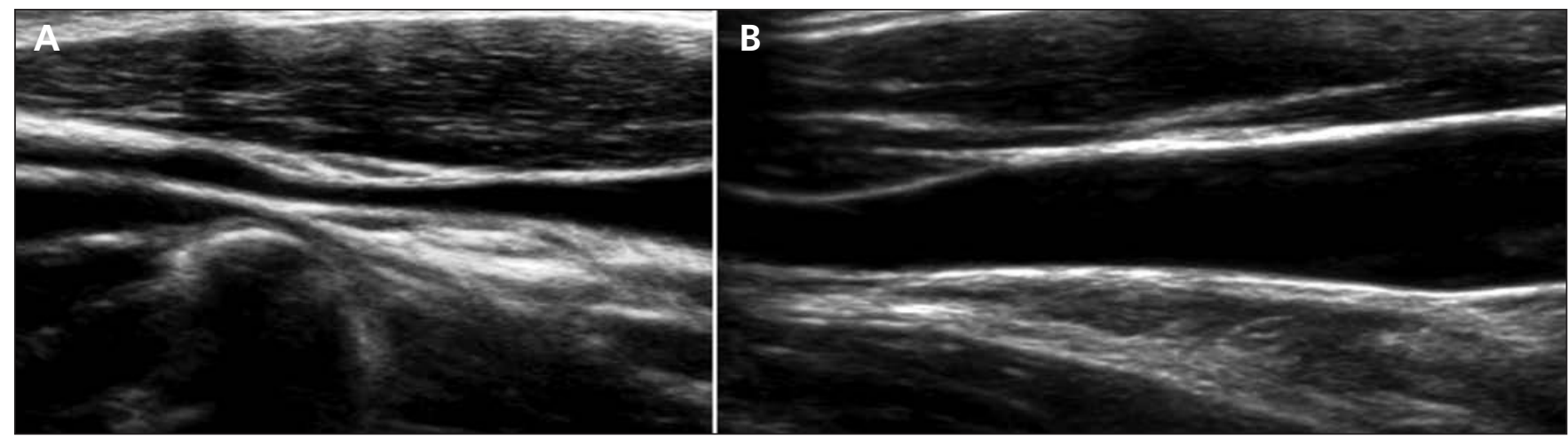

Figure 2: (A) Ultrasound image of the right internal jugular vein (sagittal plane), obtained with the participant lying supine. There is narrowing of the internal jugular vein that would be interpreted as abnormal according to the proposed ultrasound criteria for diagnosis of chronic cerebrospinal venous insufficiency. ${ }^{3,4}$ (B) Ultrasound image of the same vein obtained a few minutes later, from the same participant in the same position, shows that the vein is normal and does not have fixed narrowing. Observation of narrowing in Figure 2A may have been due to compression by the transducer or may have been secondary to a change in central venous pressure (e.g., from a change in phase of respiration or in cardiac output). 


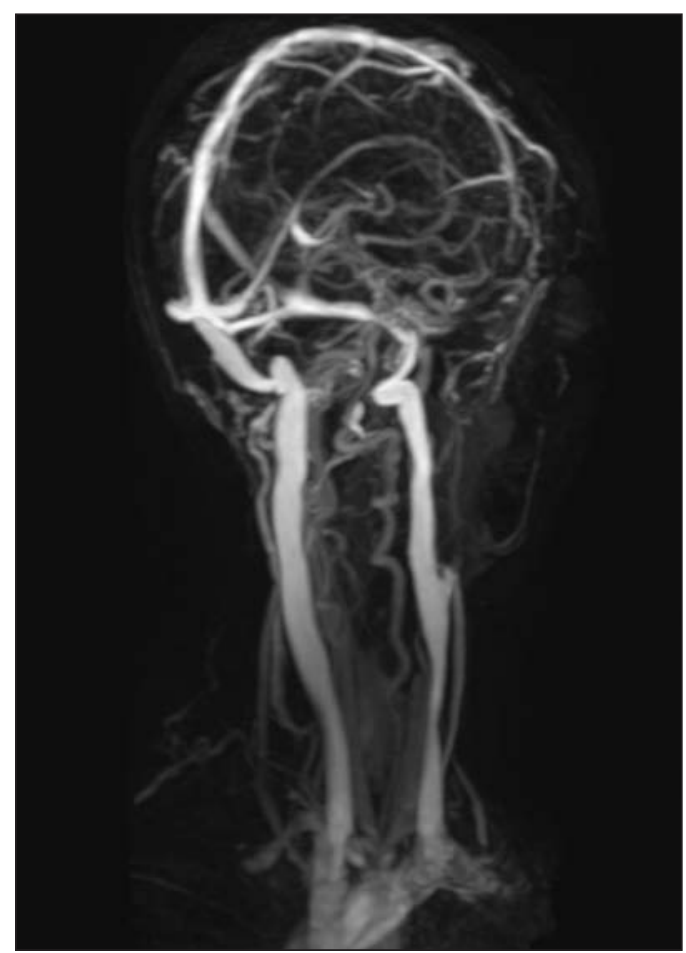

Figure 3: Gadolinium-enhanced magnetic resonance venography of a participant with $25 \%$ stenosis of the left internal jugular vein (arrow).

that the internal jugular vein is commonly partly compressed (25\% narrowing) in its extracranial course (Figure 3), and there is a tendency toward right-sided dominance for venous drainage.

Our study had several strengths, including its prospective design, complementary magnetic resonance imaging protocols and blinding strategies. Yet interpretation of our findings may be limited by failure to control for participant intravascular volume status, head position, anatomic variation of the neck strap muscles and sample size.

\section{Conclusion}

We detected no link between chronic cerebrospinal venous insufficiency and multiple sclerosis. We also identified several methodologic concerns that challenge the validity of the criteria used to define chronic cerebrospinal venous insufficiency, and in turn we dispute the authenticity of this diagnosis.

\section{References}

1. Compston A, Coles A. Multiple sclerosis. Lancet 2008;372: 1502-17.

2. Zamboni P, Galeotti R, Menegatti E, et al. A prospective openlabel study of endovascular treatment of chronic cerebrospinal venous insufficiency. J Vasc Surg 2009;50:1348-58.

3. Zamboni P, Galeotti R, Menegatti E, et al. Chronic cerebrospinal venous insufficiency in patients with multiple sclerosis. J Neurol Neurosurg Psychiatry 2009;80:392-9.

4. Zamboni P, Menegatti E, Galeotti R, et al. The value of cerebral Doppler venous haemodynamics in the assessment of multiple sclerosis. J Neurol Sci 2009;282:21-7.
5. Doepp F, Paul F, Valdueza JM, et al. No cerebrocervical venous congestion in patients with multiple sclerosis. Ann Neurol 2010; 68:173-83.

6. Baracchini C, Perini P, Calabrese M, et al. No evidence of chronic cerebrospinal venous insufficiency at multiple sclerosis onset. Ann Neurol 2011;69:90-9.

7. Baracchini C, Perini P, Causin F, et al. Progressive multiple sclerosis is not associated with chronic cerebrospinal venous insufficiency. Neurology 2011;77:844-50.

8. Tsivgoulis G, Mantatzis M, Bogiatzi C, et al. Extracranial venous hemodynamics in multiple sclerosis: a case-control study. Neurology 2011;77:1241-5.

9. Zivadinov R, Marr K, Cutter G, et al. Prevalence, sensitivity, and specificity of chronic cerebrospinal venous insufficiency in MS Neurology 2011;77:138-44

10. Bartolomei I, Salvi F, Galeotti R, et al. Hemodynamic patterns of chronic cerebrospinal venous insufficiency in multiple sclerosis. Correlation with symptoms at onset and clinical course. Int Angiol 2010;29:183-8.

11. Khan O, Filippi M, Freedman MS, et al. Chronic cerebrospinal venous insufficiency and multiple sclerosis. Ann Neurol 2010; 67:286-90.

12. Worthington V, Kilestein J, Eikelenboom MJ, et al. Normal CSF ferritin levels in MS suggest against etiologic role of chronic venous insufficiency. Neurology 2010;75:1617-22.

13. Mayer CA, Pfeilschifter W, Lorenz MW, et al. The perfect crime? Chronic cerebrospinal venous insufficiency not leaving a trace in MS. J Neurol Neurosurg Psychiatry 2011;82:436-40.

14. Simka M, Kostecki J, Zaniewski M, et al. Extracranial Doppler sonographic criteria of chronic cerebrospinal venous insufficiency in the patients with multiple sclerosis. Int Angiol 2010; 29:109-14.

15. Menegatti E, Genova V, Tessari M, et al. The reproducibility of colour Doppler in chronic cerebrospinal venous insufficiency associated with multiple sclerosis. Int Angiol 2010;29:121-6.

16. Yamout B, Herlopian A, Issa Z, et al. Extracranial venous stenosis is an unlikely cause of multiple sclerosis. Mult Scler 2010;16:341-8.

17. Baracchini C, Valdueza JM, Del Sette M, et al. CCSVI and MS: a statement from the European Society of Neurosonology and Cerebral Hemodynamics. J Neurol 2012;259:2585-9.

18. Comi G, Battaglia MA, Bertolotto A, et al.; CoSMo Collaborative Study Group. Observational case-control study of the prevalence of chronic cerebrospinal venous insufficiency in multiple sclerosis: results from the CoSMo study. Mult Scler 2013;19:1508-17.

19. Leone MA, Raymkulova O, Naldi P, et al. Chronic cerebrospinal venous insufficiency is not associated with multiple sclerosis and its severity: a blind-verified study. PLOS ONE 2013;8: e56031.

20. Valdueza JM, Florian Doepp F, Schreiber SJ, et al. What went wrong? The flawed concept of cerebrospinal venous insufficiency. J Cereb Blood Flow Metab 2013;33:657-68.

21. Barreto AD, Brod SA, Bu TT, et al. Chronic cerebrospinal venous insufficiency: case-control neurosonography results. Ann Neurol 2013;73:721-8.

22. Singh AV, Zamboni P. Anomalous venous blood flow and iron deposition in multiple sclerosis. J Cereb Blood Flow Metab 2009; 29:1867-78

23. Zivadinov R, Galeotti R, Hojnacki D, et al. Value of MR venography for detection of internal jugular vein anomalies in multiple sclerosis: a pilot longitudinal study. AJNR Am J Neuroradiol 2011; 32:938-46.

24. Pullman D, Zarzeczny A, Picard A. "Media, politics and science policy: MS and evidence from the CCSVI trenches." BMC Med Ethics 2013;14:6.

25. McTaggart RA, Fischbein NJ, Elkins CJ, et al. Extracranial venous drainage patterns in patients with multiple sclerosis and healthy controls. AJNR Am J Neuroradiol 2012;33:1615-20.

26. Laupacis A, Lillie E, Dueck A, et al. Association between chronic cerebrospinal venous insufficiency and multiple sclerosis: a meta-analysis. CMAJ 2011;183:E1203-12.

27. Tsivgoulis G, Sergentanis TN, Chan A, et al. Chronic cerebrospinal venous insufficiency and multiple sclerosis: a comprehensive metaanalysis of case controlled studies. Ther Adv Neurol Disord 2014; 7:114-36.

28. Zwischenberger BA, Beasley MM, Davenport DL, et al. Metaanalysis of the correlation between chronic cerebrospinal venous insufficiency and multiple sclerosis. Vasc Endovascular Surg 2013;47:620-4.

29. Polman $\mathrm{CH}$, Reingold SC, Banwell B, et al. Diagnostic criteria for multiple sclerosis: 2010 revisions to the McDonald criteria. Ann Neurol 2011;69:292-302.

30. Poser CM, Paty DW, Sheinberg L, et al. New diagnostic criteria for multiple sclerosis: guidelines for research protocols. Ann Neurol 1983;13:227-31. 
31. Lublin FD, Reingold SC. Defining the clinical course of multiple sclerosis: results of an international survey. National Multiple Sclerosis Society (USA) Advisory Committee on Clinical Trials of New Agents in Multiple Sclerosis. Neurology 1996;46: 907-11.

32. Lennon VA, Wingerchuk DM, Kryzer TJ, et al. A serum autoantibody marker of neuromyelitis optica: distinction from multiple sclerosis. Lancet 2004;364:2106-12.

33. Kurtzke JF. Rating neurological impairment in multiple sclerosis: an expanded disability status scale (EDSS). Neurology 1983; 33:1444-52.

34. Cerebral venous function and anomaly CME program. Buffalo (NY): Buffalo Neuroimaging Analysis Center. Available: www.bnac.net/?page_id=607 (accessed 2014 May 22).

35. Nedelmann M, Eicke BM, Dieterich M. Functional and morphological criteria of internal jugular valve insufficiency as assessed by ultrasound. J Neuroimaging 2005;15:70-5.

36. Lichtenstein D, Saifi R, Augarde R, et al. The internal jugular veins are asymmetric. Usefulness of ultrasound before catheterization. Intensive Care Med 2001;27:301-5.

37. Weir B. Multiple sclerosis — a vascular etiology? Can J Neurol Sci 2010;37:745-57.

Competing interests: Fiona Costello has received funding support for steering committee participation from Novartis, for advisory board participation from Questcor, Allergan, Roche and Celgene, and for educational teaching from EMD Serono; she has also received study sponsorship from Biogen Idec and Novartis. Richard Frayne is co-inventor of the Time-Resolved Imaging of Contrast Kinetics (TRICKS) technique used for magnetic resonance venography in this study; he receives royalty payments from the Wisconsin Alumni Research Foundation for licensing of this technology. He also receives support from General Electric Healthcare (through Alberta Health Services) for other magnetic resonance imaging research. Michael Hill has received payment for board membership from the Heart and Stroke Foundation (Alberta, NWT \& Nunavut), the Institute for Circulatory and Respiratory Health (Canadian Institutes for Health
Research) and the Canadian Federation of Neurological Sciences; consultancy payments from Vernalis Group and Merck Co.; grants for other studies from Hoffmann-La Roche Canada and Covidien; and and payment for lectures from Hoffmann-La Roche Canada, Servier Canada and BMS Canada. He owns stock in Calgary Scientific Inc., a technology software company (for imaging unrelated to the current study) and has received salary support from the Heart and Stroke Foundation (Alberta, NWT \& Nunavut) and Alberta Innovates Health Solutions. No other competing interests were declared.

Affiliations: Departments of Clinical Neurosciences (Costello, Modi, Scott, Davenport, Trufyn, Frayne, Ciura, Goyal, Hill), Surgery (Costello), Radiology (Modi, Lautner, Bhayana, Scott, Frayne, Ciura, Goyal), Pediatrics (Mah), Medical Genetics (Davenport), Medicine (Hill) and Community Health Sciences (Hill); Hotchkiss Brain Institute (Costello, Davenport, Hill); and Seaman Family Centre, Foothills Medical Centre, Alberta Health Services (Bhayana, Scott, Frayne, Goyal), University of Calgary, Calgary, Alta.

Contributors: Fiona Costello, Jayesh Modi, David Lautner, Deepak Bhayana, James N. Scott, Richard Frayne, Mayank Goyal and Michael Hill contributed substantially to conception and design of the study and to acquisition, analysis and interpretation of the data. Jessie Trufyn, W. Jeptha Davenport, Viesha A. Ciura and Jean Mah contributed substantially to acquisition of data. All authors drafted the article and revised it critically for important intellectual content, gave final approval of the version to be published and agree to act as guarantors of the work.

Funding: This study was funded by the Multiple Sclerosis Society of Canada. The study sponsor had no role in the design of the study, the collection, analysis or interpretation of the data, the writing of the report or the decision to submit the manuscript for publication. 